\title{
Blurring the boundaries
}

\section{A touring exhibition shows the value of the Swiss 'artists-in-labs' programme, find Deborah Dixon, Harriet Hawkins and Mrill Ingram.}

$\mathrm{W}$ ith sci-art collaborations on the rise, this refreshing exhibition stands out as a reflection on how both parties can benefit from the tensions and challenges in such meetings of minds. Think Art - Act Science is the first exhibition to showcase art that has come out of the Swiss 'artists-in-labs' programme, a joint project of the Institute for Cultural Studies in the Arts at the Zurich University of the Arts and the federal culture ministry.

Since 2003, 28 artists, most from Switzerland, have been placed in labs there and, since 2009, in China. This exhibition of 'prototypes' by nine of them looks at how interdisciplinary collaborations work, and how the ideas they spark can best be conveyed to the public. Showing at Arts Santa Monica in Barcelona, Spain, until 15 May, the exhibition then tours several US cities and Ireland.

Some installations capture the artists' visceral response to their scientific engagements. Sylvia Hostettler's polyester resin sculptures (Regeneration I and II, 2008), their protuberances glowing with malign intensity, suggest the monstrous nature of the phenomena she encountered in the Center for Integrative Genomics at the University of Lausanne. Equally unsettling are Pe Lang's Kinetic Speakers, a cluster of sleek, swivelling microphonecum-loudspeakers that simultaneously listen and react to sound. These finely crafted electronic sculptures, made in collaboration with the Swiss Center for Electronics and Microtechnology, Alpnach, dispel notions of

media technologies as passive.

That the works are prototypes is significant - the programme stresses the collaborative production of ideas over a finished output. Each work is accompanied by a video in which the artists and scientists convey the ways in which communication did and did not take place, and discuss how the works evolved. These videos reveal how all involved had their expectations shaken.

Scientists at the Swiss Federal Institute of Aquatic Science and Technology in Dübendorf, for example, which hosted artist Ping Qui, expressed surprise that her work, like their research, privileges precision and rigour. In her Breathing Plants (2008), tumescent plastic sculptures resembling roots grow and shrink in response to the humidity in a room, accompanied by recorded sounds of human inhalation and exhalation.

Also on display is Christian Gonzenbach's light sculpture QUARC, Quantum Art Crystal (2009), which emerged from his residency at the University of Geneva physics department and CERN, where he came to admire the aesthetic sensibility and craft of scientists there.

\section{ONATURE.COM}

Martin Kemp on neuroscience and art: go.nature.com/dskge?

\section{A response to experi-} ments on dark matter and complex systems, QUARC deploys a crystalline lattice of neon tubes that flash on and off in response to unpredictable movements of a series of small magnets. Meanwhile, the video of scientists at the Artificial Intelligence Laboratory, University of Zurich, shows them enthused by the challenge of creating the dancing robot envisioned by choreographer Pablo Ventura.

The videos touch on often-overlooked aspects of collaboration, such as alienation and miscommunication. Wenfeng Liao, for instance, former artist-in-residence at the Swiss Federal Institute of Forest, Snow and Landscape Research in Birmensdorf, spoke of finding lab science strange. His point of engagement was the researchers' office plants. His photo Office Plants (2010), for instance, which shows snapshots of the plants nailed to the trees the scientists study, led to a discussion about artificial boundaries between living and working environments and those serving as research subjects.

This contemplative exhibition demonstrates, with considerable style, the value of sci-art collaboration in opening up productive spaces of mutual engagement, inspiration and intrigue.

Deborah Dixon and Harriet Hawkins are based at the Institute of Geography and Earth Sciences, Aberystwyth University, UK. Mrill Ingram is based at the Department of Geography, University of WisconsinMadison, USA. e-mail:hah7@aber.ac.uk

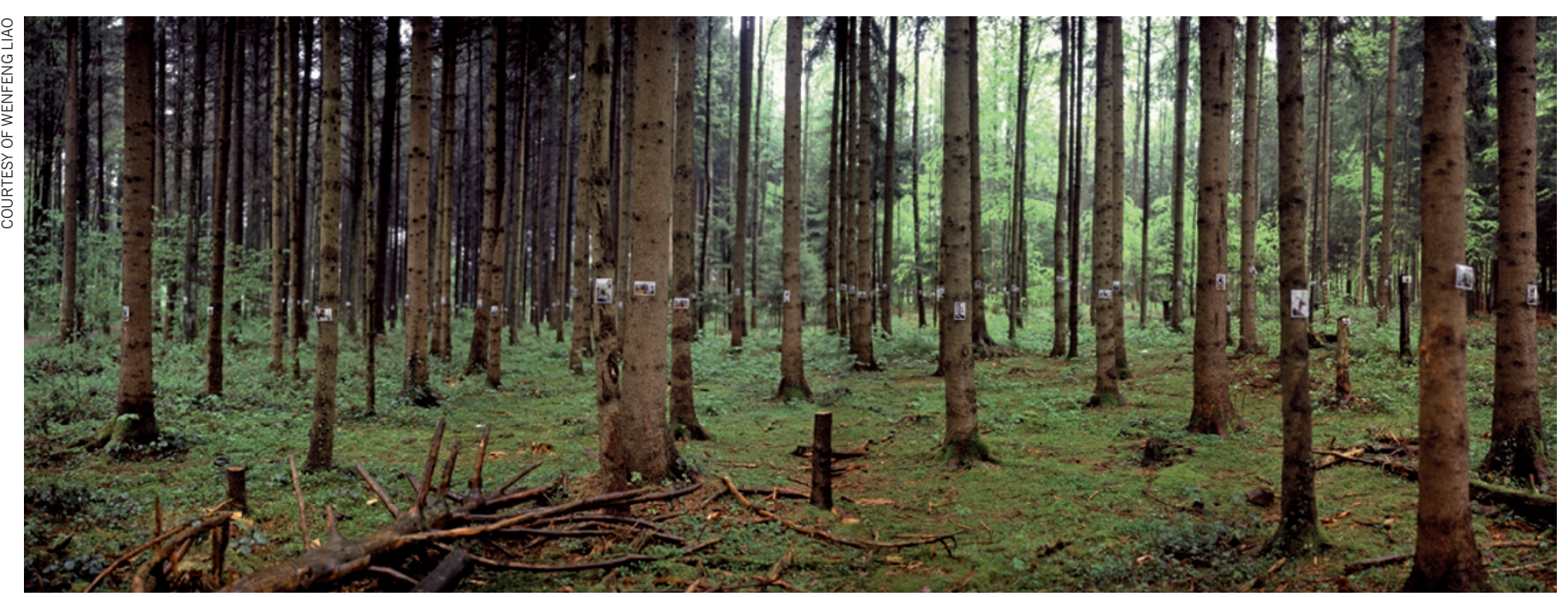

Small Paths (2), a video installation by Wenfeng Liao, former artist-in-residence at the Swiss Federal Institute of Forest, Snow and Landscape Research. 